\title{
LAS SOCIEDADES DE GESTIÓN COLECTIVA FRENTE A LA LIBRE COMPETENCIA ECONÓMICA
}

FELIPE ABELlo MONSALVO*

\section{INTRODUCCIÓN}

La gestión del derecho de autor se presenta como un mecanismo regulado por ley para la administración del recaudo por el uso de derechos patrimoniales de diversos titulares de derechos de autor, a través de un agente denominado sociedad de gestión colectiva (en adelante, SGC) que actúa como mandatario de los titulares de los mencionados derechos.

Las SGC se configuran como un agente intermediario entre el titular de la obra y el usuario (consumidor), al otorgar directamente las licencias que autorizan el uso de las obras y, a su vez, remunerar a los titulares por dicho uso.

De esta intermediación resultan eficiencias para los participantes en el mercado: por una parte, un agente centraliza la representación de un mayor número de titulares, lo que permite otorgar licencias que den acceso a la explotación de un mayor número de obras; por otra, este agente realiza la gestión de los derechos de una colectividad, reduciendo los costos legales y de comercialización en que tendría que incurrir cada titular al gestionar directamente sus derechos.

Las mencionadas eficiencias generadas por las SGC facilitan los mecanismos de transacción; ejemplo de ello son las licencias o autorizaciones generales que disminuyen el costo de vigilancia y los costos de transacción.

A las referidas eficiencias de las SGC se suma una característica conferida a ellas por parte de la doctrina, las autoridades de competencia y los organismos encargados de la promoción y protección de la propiedad intelectual a nivel mundial,

* Abogado de la Pontificia Universidad Javeriana, profesor de Derecho de Contratos de la Universidad del Sinú, profesor de Derecho de los Negocios de la Pontificia Universidad Javeriana y candidato a maestro en Propiedad Intelectual de la Universidad Externado de Colombia (Bogotá, Colombia). Contacto: felipe.abello@est.uexternado.edu.co / felipeabellom@ gmail.com Fecha de recepción: 7 de mayo de 2017. Fecha de aceptación: 30 de mayo de 2017. Para citar el artículo: Abello-Monsalvo, F. "Las sociedades de gestión colectiva frente a la libre competencia económica", Revista La Propiedad Inmaterial n. ${ }^{\circ}$ 23, Universidad Externado de Colombia, enero-junio 2017, pp. 131-146. Dor: https://doi.org/10.18601/16571959.n23.05 
que consiste en que, bajo determinadas condiciones, la gestión colectiva de los derechos de autor resulta indispensable.

Estas dos particularidades -eficiencia e indispensabilidad-ubican a las SGC en una posición privilegiada en el mercado relevante ${ }^{1}$ en el que actúan. Privilegio que, dependiendo del estudio de mercado para cada caso específico, puede convertirse en una posición de dominio por parte de las SGC y, en consecuencia, el régimen de protección de la competencia ${ }^{2}$ exigirá más respecto de las conductas a desarrollar por las SGC como agentes en ese determinado mercado.

Por lo anterior y teniendo en cuenta que la posición de dominio en Colombia no es reprochable per se, sino que lo que la convierte en sancionable es su abuso, resulta necesario proponer unos lineamientos mínimos para que las SGC en el desarrollo de su gestión no afecten la libre competencia económica con una práctica restrictiva de la competencia, específicamente, con un abuso de posición de domino establecido en los términos del artículo 50 del Decreto 2153 de 1992.

\section{LA GESTIÓN COLECTIVA EN CABEZA DE LAS SOCIEDADES DE GESTIÓN COLECTIVA}

La gestión colectiva se refiere al conjunto de actividades que permiten al titular materializar las prerrogativas otorgadas por el derecho de autor para explotar su obra. En efecto,

... las actividades en que se sustancia la gestión son, básicamente, las de vigilar la explotación de las obras y prestaciones administrativas, negociar con los usuarios eventuales, otorgarles autorizaciones o licencias bajo remuneración, recaudar las remuneraciones convenidas (en esas autorizaciones) y satisfacer a los correspondientes titulares de las obras o prestaciones explotadas, previa distribución (reparto) cuando esas remuneraciones provengan de explotaciones autorizadas que comprendan obras o prestaciones de diferentes titulares. (De las entidades de gestión de los derechos reconocidos en la ley, 1995).

La jurisprudencia constitucional ha establecido que el derecho que el titular ejerce sobre su obra, que es considerado "una forma de propiedad" (Sentencia C-509 de

1 Para cada caso en concreto el mercado dependerá de la modalidad del derecho patrimonial que se explote por parte de las SGC. Para el ejemplo, en el caso sAYCo el mercado relevante sobre el que se investigó fue el derecho patrimonial de comunicación pública (Superintendencia de Industria y Comercio, 2016).

2 Así se refirió la Corte Constitucional, en sentencia C-032 de 25 de enero de 2017, a las reglas de procedimiento para las investigaciones adelantadas por la sic, bajo el régimen de protección de la competencia que se encuentra reglado en el Decreto 2153 de 1992, la Ley 1340 de 2009 y el Decreto 019 de 2012, contando con las cláusulas de integración de la Ley 1437 de 2011 que contiene el Código de Procedimiento Administrativo y de lo Contencioso Administrativo. 
2004), puede gestionarse tanto de manera individual como de forma colectiva ${ }^{3}$. $\mathrm{Al}$ respecto ha afirmado:

En el ordenamiento jurídico colombiano, el Legislador faculta a los autores y/o titulares de obras literarias y artísticas para autorizar de manera previa y expresa la utilización de sus creaciones. Como desarrollo de dicha atribución, se ha previsto que el recaudo de los derechos de autor y sus derechos conexos se realice de manera directa a través de la gestión individual de cada interesado o mediante la gestión colectiva realizada por personas jurídicas instituidas para dicho efecto, denominadas sociedades de gestión colectiva. (Sentencia C-124 de 2013).

Así las cosas, es necesario hacer referencia a las características propias de cada una de esas modalidades de gestión de los derechos de autor.

\section{I.I. Gestión individual}

La gestión individual es la administración de los derechos de autor de contenido patrimonial realizada directamente por el titular. Se refiere al ejercicio natural de las prerrogativas que mediante los artículos 13 de la Decisión 351 de 1993 y 12 de la Ley 23 de 1982 se otorgan al titular de la obra. Tanto la que realiza el titular directamente como aquella que adelanta a través de formas asociativas distintas a la gestión colectiva asociativas distintas a la gestión colectiva.

En relación con las actividades concretas que esta modalidad de gestión comprende, la doctrina ha precisado que incluye dos: de un lado, la de "negociar con el usuario las condiciones de la autorización y conferirla" (Derecho de autor y derechos conexos, 2006), y del otro, "efectuar la recaudación" (Derecho de autor y derechos conexos, 2006) correspondiente.

Existen ciertos modalidades de uso de los derechos patrimoniales de autor, a saber, comunicación al público en establecimientos abiertos al público y/o comunicación al público en conciertos (Superintendencia de Industria y Comercio, 2016) que no sería eficiente realizar a través de este modelo de gestión. En consecuencia, para ciertos usos la gestión colectiva es indispensable.

\section{I.2. Gestión COLECtiva}

La gestión colectiva se define como:

... el sistema de administración de derechos de autor y de derechos conexos por el cual sus titulares delegan en organizaciones creadas al efecto la negociación de las condicio- 
nes en que sus obras, sus prestaciones artísticas o sus aportaciones industriales -según el caso- serán utilizadas por los difusores y otros usuarios primarios, el otorgamiento de las respectivas autorizaciones, el control de las utilizaciones, la recaudación de las remuneraciones devengadas y su distribución o reparto entre los beneficiarios (Derecho de autor y derechos conexos, 2006).

En el contexto colombiano, el marco jurídico de la gestión colectiva se encuentra establecido en el capítulo xi de la Decisión 351 de 1993, el capítulo Xvi de la Ley 23 de 1982, el capítulo in de la Ley 44 de 1993 y el Decreto 3942 de 2010. La normativa citada permite aclarar que la gestión colectiva se desarrolla a través de personas jurídicas que reciben de un número representativo de titulares de derechos de autor el encargo de gestionar sus derechos de contenido patrimonial y de actuar en su representación con ese propósito ${ }^{4}$.

La gestión colectiva se organizó en Colombia alrededor de las siguientes características: su carácter privado, la ausencia de un ánimo de lucro, su función como recaudadoras de los valores que generan las explotaciones o utilizaciones de las obras, y el objetivo de distribuir el recaudo entre los titulares de los derechos patrimoniales de las obras (Dirección Nacional de Derecho de Autor, 2007).

A diferencia de otros ordenamientos -como los de Paraguay o España-, en el de Colombia no existe dentro del régimen de protección de derechos de autor ninguna norma que establezca la gestión colectiva como obligatoria.

En Colombia se reconoce la libertad del titular para gestionar sus derechos de manera individual o colectiva. Con fundamento en la jurisprudencia constitucional ${ }^{5}$, los titulares de derechos de autor están habilitados para emplear formas de asociación distintas a las propias de la gestión colectiva para efectos de gestionar de manera conjunta sus derechos.

\footnotetext{
4 En la actualidad, las únicas sociedades de gestión colectiva con personería jurídica y autorización de funcionamiento otorgadas por la Dirección Nacional de Derechos de Autor, y por consiguiente legitimadas para gestionar y recaudar colectivamente los derechos de autor y conexos, según se trate, son: (i) Sociedad de Autores y Compositores de Colombia, sAYCO, con autorización de funcionamiento conferida mediante Resolución 070 del 5 de junio de 1997; (ii) Asociación Colombiana de Intérpretes y Productores Fonográficos, ACINPRO, con autorización de funcionamiento conferida mediante Resolución 125 del 5 de agosto de 1997; (iii) ACTOREs Sociedad Colombiana de Gestión, con personería jurídica reconocida y confirmada mediante las resoluciones 028 del 29 de noviembre de 1989 y 018 del 21 de febrero de 1997, respectivamente; (iv) Centro Colombiano de Derechos Reprográficos, CDR, con personería jurídica reconocida mediante Resolución 088 del 14 de julio de 2000 y autorización de funcionamiento concedida mediante Resolución 035 del 18 de febrero de 2002. y (iv) Entidad de Gestión de Derechos de los Productores Audiovisuales de Colombia, EGEDA Colombia, con personería jurídica reconocida mediante Resolución 232 del 28 de noviembre de 2005 y autorización de funcionamiento concedida mediante Resolución 208 del 16 de noviembre de 2006.

5 Así se ha referido la Corte Constitucional en distintas sentencias. Para el ejemplo: sentencias C-519 de 1999, C-509 de 2004, C-424 de 2005, C-1118 de 2005, C-833 de 2007 y C-912 de 2011, entre otras. Véase también Ficsor, Mihály, La gestión colectiva del derecho de autor y los derechos conexos, Ginebra, омрі, 2002, pp. 12 у 13.
} 
Los agentes que intervienen en la gestión del derecho de autor ${ }^{6}$ son: por una parte, los titulares de derechos de autor, a quienes se les ofrece el servicio de gestión $y$, una vez efectuado el recaudo, se les distribuyen los ingresos percibidos por la utilización de las obras; y, por otra, los usuarios de las obras, con quienes se desarrolla la gestión correspondiente mediante la negociación de las condiciones de uso, la concesión de la autorización necesaria y la ejecución de las labores de recaudo.

La doctrina especializada, así como las autoridades de competencia y los organismos encargados de la promoción y protección de la propiedad intelectual a nivel mundial ${ }^{7}$, han dejado claro que, bajo determinadas condiciones, la gestión colectiva de los derechos de autor resulta indispensable. "La gestión de algunas formas de utilización de obras protegidas por el derecho de autor solo puede llevarse a cabo -eficientemente- mediante una sociedad de gestión colectiva" (Superintendencia de Industria y Comercio, 2016).

Sobre el particular se ha dicho que "la razón por la que muchas veces los derechos de autor $[\ldots]$ no pueden ejercerse individualmente es que las obras de que se trata son utilizadas por un gran número de usuarios, en lugares distintos y en diferentes momentos. Los particulares, en general, carecen de capacidad para controlar todas esas utilizaciones, negociar con los usuarios y recaudar las remuneraciones" ( $\mathrm{La}$ gestión colectiva del derecho de autor y los derechos conexos, 2002).

Este escrito se centra en las personas jurídicas que han sido instituidas con el fin de recaudar los derechos de autor y sus derechos conexos, y no en las dificultades propias que enfrenta la gestión individual de los derechos de autor ${ }^{8}$. Por lo anterior, a continuación se presenta un acercamiento a la gestión que realizan las SGC.

\section{Sociedades De gestión Colectiva}

Las SGC han sido las encargadas de realizar las actividades que permiten al titular de una obra materializar las prerrogativas otorgadas por el derecho de autor para explotar su obra. En ese sentido, el titular ha confiado a las SGC la administración y explotación de su obra?

6 Puede haber otros agentes involucrados, dependiendo de la forma en la que el titular decida gestionar su derecho de autor. La gestión del derecho de autor se puede realizar a través de una SGC, de manera conjunta y/o individualmente por parte del titular del derecho de autor. Cuando se realiza con la ayuda de una sGC, esta será un agente fundamental dentro de la gestión.

7 En ese sentido lo ha reiterado la Comisión Nacional de la Competencia de España. Informe sobre la gestión colectiva de derechos de propiedad intelectual. 2009, pp. 24 ss. Y véase Dirección Nacional de Derecho de Autor, Manual de Derecho de Autor para Alcaldias y Gobernaciones, 2011, p. 50 (fol. 48 del cdno. público n.o 1).

8 Es de resaltar que el bloqueo de la gestión individual también puede constituirse en una práctica restrictiva de la competencia.

9 Adicionalmente a las SGC registradas debidamente en la Dirección Nacional de Derechos de Autor, existe otro grupo de asociaciones que también gestionan determinados derechos patrimoniales de autor; es el caso de la Asociación para la Protección de los Derechos Intelectuales sobre Fonogramas y Videogramas Musicales (APDIF). 
Las SGC encuentran fundamento en el derecho de asociación consagrado en el artículo 38 de la Constitución Política (Sentencia C-124 de 2013). La función de estas personas jurídicas consiste en negociar con los potenciales usuarios de las obras incluidas en su repertorio las condiciones de utilización correspondientes -es decir, la forma en que se llevará a cabo el uso y la remuneración que se seguirá de ello- y, acordadas esas condiciones, conceder a los usuarios la autorización que legitima la utilización de la obra.

Además, las SGC se encargan de: (i) monitorear la utilización de las obras cuya gestión se les encomendó; (ii) recaudar las remuneraciones pactadas de las personas que hayan usado su repertorio y, finalmente, (iii) distribuir, entre los titulares de derechos de autor cuyos derechos administran, las regalías obtenidas sobre la base de algún criterio de distribución establecido para el efecto.

Cada SGC tiene el deber de administrar el repertorio que representa de acuerdo con los mandatos que le son dados por sus asociados y representados. Adicionalmente, atendiendo a los contratos recíprocos que tienen las SGC con otras sociedades a nivel mundial ${ }^{10}$.

Las SGC tienen una presunción legal para la mencionada representación en términos del catálogo (repertorio) objeto de cobro, que consiste en que sin necesidad de probar el mismo puedan recaudar con una determinación específica de los derechos que se gestionan, al igual que sobre las diferentes modalidades que son objeto de explotación por parte de la sociedad.

Es importante resaltar que cada una de estas sociedades tiene un mercado específico en función del derecho patrimonial que gestionan. Es así como para un derecho patrimonial como el de comunicación pública de autores y compositores solo existe SAYCO y para los derechos conexos ACINPRO ${ }^{11}$.

Por lo anterior, en la actualidad en Colombia no existen dos sociedades de gestión colectiva que administren el mismo derecho patrimonial para el mismo titular.

10 Estos contratos de representación recíproca son usuales entre las SGC de diferentes países y consisten en que el recaudo de la utilización del repertorio administrado por cada SGC en otro país sea cobrado por la sociedad con la que se tiene el convenio y de igual forma recíprocamente. La Confederación Internacional de Sociedades de Autores y Compositores (CISAC) es una organización internacional no gubernamental, sin fines de lucro, que agrupa entidades de gestión colectiva de derechos de autor de varios países. Fue fundada en 1926-1927 en París -donde tiene aún su domicilio. Esta entidad emite directrices sobre cómo deben funcionar las SGC, en líneas generales, y coordina también los esfuerzos necesarios para integrar los principios y los métodos de la administración colectiva, sin interferir en la organización interna de cada sociedad.

11 En ese sentido, el artículo 30 de la Ley 44 de 1993 reza: "Las sociedades de gestión colectiva de derechos de autor y derechos conexos quedan obligadas a elaborar reglamentos internos en los que se precise la forma como deberá efectuarse entre los socios el reparto equitativo de las remuneraciones recaudadas, así como la forma como se fijarán las tarifas por concepto de las diversas utilizaciones de las obras, prestaciones artísticas y de las copias o reproducciones de fonogramas". Así las cosas, la normativa aplicable a la gestión colectiva faculta a este tipo de sociedades para fijar, mediante reglamentos internos, las tarifas que cobrarán por la utilización de las obras que hacen parte de su repertorio. 
Una vez descritas las funciones de las SGC, es importante establecer las eficiencias que se generan para el titular de la obra al realizar la gestión de sus derechos de autor a través de estas personas jurídicas.

\section{EFICIENCIAS E INDisPENSABILIDAD - VENTAJAS DE LAS SOCIEDADES DE GESTIÓN COLECTIVA}

Aunque en Colombia no existe ninguna prohibición legal que impida la formación de otras sociedades para la gestión colectiva de cada mercado que las habilitan, entre otras cosas para "autorizar genéricamente el uso de obras de las que no son titulares" y "realizar el recaudo de tarifas distintas de aquellas que voluntariamente se hayan convenido con los usuarios por la explotación de los derechos de los que son titulares" (Sentencia C-833 de 2007), las sGC gozan de prerrogativas específicas de las que carece la gestión individual ${ }^{12}$. Ejemplo de estas ventajas se encuentra en la denominada legitimación presunta, prevista, según la Dirección Nacional de Derechos de Autor, en los artículos 49 de la Decisión 351 de 1993 y 9 del Decreto 3942 de 2010.

Las razones económicas que justifican la creación y la función de las sociedades de gestión colectiva (Comisión Nacional de la Compentencia de España, 2009) son fuentes que evidencian que la justificación económica de la gestión colectiva consiste en la disminución de, entre otros, tres tipos de costos asociados al ejercicio de los derechos de autor de contenido patrimonial (Merges, 1996). Veamos.

\section{I. LOS COSTOS DE BÚSQUEDA}

La identificación de los oferentes y demandantes de las obras protegidas por el derecho de autor puede ser costosa (Supreme Court of the United States, 1979). Desde la perspectiva del titular, porque sin gestión colectiva tendría que identificar los usuarios potenciales para dirigir hacia ellos la labor de promoción de sus obras. Desde la del usuario, porque en esas condiciones él tendría la carga de establecer quiénes son todos los titulares de las obras que pretende utilizar, lo que se torna más complicado en el caso de obras cuyos derechos pertenezcan a varios titulares (Análisis económico de la gestión del derecho de autor, 2011). La gestión colectiva permite que una persona jurídica centralice la información necesaria para ese contacto entre oferentes y demandantes, con lo que posibilita la negociación correspondiente (Competition policy and its implications for intellectual property rigths in the United States, 2007). 


\subsection{Los COSTOS DE NEGOCIACIÓN}

Acerca de los costos de negociación, la ausencia de un sistema de gestión colectiva supone que para cada operación cada uno de los miles de titulares de derechos de autor deba negociar las condiciones de utilización correspondientes a cada una de sus obras con cada uno de los usuarios interesados en utilizarlas, con el agravante de que esos titulares no necesariamente tienen los elementos de juicio suficientes para establecer la remuneración que corresponde por el uso de sus obras (United States District Cout, 2014).

Ante ese panorama se ha considerado que, dado el enorme número de negociaciones bilaterales que serían necesarias para legitimar el uso de las obras, un sistema de gestión colectiva se presenta como la única solución razonable (Contracting into Liability rules: Intellectual Property Rights and Collective Rights Organizations, 1996).

La conclusión anterior se fundamenta en que, además del carácter profesional de la SGC -que, entre otras cosas, le permite establecer tarifas adecuadas a las condiciones del mercado-, esas instituciones cuentan con mecanismos que reducen los costos asociados a la determinación de las condiciones de uso de las obras que administran, entre los que se encuentran la posibilidad de establecer las tarifas correspondientes (art. 30 Ley 44 de 1993) y la de usar las denominadas licencias generales -blanket licenses-, que habilitan a cada usuario la utilización de todo el repertorio administrado por la sociedad de gestión colectiva.

\subsection{Los COSTOS DE EJECUCión DEL CONTRATO}

Debe decirse que la inexistencia de un sistema de gestión colectiva impondría al usuario la tarea de monitorear la utilización de su obra para, de un lado, verificar que se use de conformidad con lo pactado y, del otro, calcular la tarifa en caso de que esté determinada por la cantidad de usos ${ }^{13}$.

Dado que las sociedades de gestión colectiva actúan en representación de todos sus miembros en la medida en que centralizan la ejecución de las funciones que se viene comentando, están en condiciones de generar economías de escala tanto en la labor de monitoreo de la utilización de las obras de su repertorio -con la finalidad de identificar los usos no autorizados y determinar las condiciones para la distribución de las regalías- como en la labor de recaudo correspondiente.

13 El usuario tendría que asumir los costos asociados a la actividad de recaudación que, por ejemplo, en el caso de la comunicación pública en establecimiento abierto al público supondría estructurar algún mecanismo que permitiera determinar la cantidad de usos que ha tenido su obra y recorrer todo el territorio nacional para hacer el cobro. 


\subsection{Gestión colectiva como indispensable}

Sobre la base de lo expuesto, se ha considerado que la gestión individual es factible $-\mathrm{y}$, por lo tanto, que la colectiva no es indispensable- si el número de usuarios es reducido, existe un esquema de licenciamiento en el que converge un número también reducido de titulares, es factible la localización de unos y otros agentes y, por último, existe certidumbre sobre la utilización de la obra (Comisión Nacional de la Compentencia de España, 2009).

En el mismo sentido, se ha considerado que la gestión colectiva es indispensable en aquellos casos en que el número de titulares y usuarios es alto, su localización es difícil y existen dificultades para establecer con certeza la utilización de la obra y las condiciones en que se presentó.

Expuestos los fundamentos de las eficiencias y el carácter indispensable de las SGC para ciertas modalidades de explotación de los derechos patrimoniales de autor, a continuación se presenta una descripción de la posición de dominio por parte de un agente en un determinado mercado para demostrar que las SGC ostentan posición de dominio en ciertas modalidades de utilización del derecho patrimonial.

\section{LA POSICIÓN DE DOMINIO POR PARTE DE LAS SOCIEDADES DE GESTIÓN COLECTIVA}

Para demostrar que las SGC ostentan posición de dominio en los mercados en los que su gestión es indispensable se hace necesario presentar una descripción de la evolución del concepto de dominancia.

Dentro de las varias concepciones que pueden admitirse en relación con la definición de la institución de dominancia (The Concept of Dominance in Article 82, 2006) (Dominant Position: A Term in Search of Meaning, 2014), la jurisprudencia internacional ha considerado que consiste en una posición de fortaleza económica que habilita a un agente para prevenir que se mantenga una competencia efectiva (1978), posibilidad que surge debido a que ese agente tiene las condiciones para comportarse de manera independiente respecto de sus competidores, compradores y consumidores ${ }^{14}$.

$14 \mathrm{La}$ independencia con la que puede actuar un agente se ha reiterado en distintos pronunciamientos; para el ejemplo: (i) Sentencia del Tribunal de Justicia de las Comunidades Europeas. Decisión de 14 de febrero de 1978. Asunto 27/26. United Brands Company and United Brands Continental BV v. Commission of the European Communities; (ii) Sentencia del Tribunal de Justicia de las Comunidades Europeas. Decisión de 13 de febrero de 1979. Asunto 85/76. Hoffmann-La Roche \& Co. AG v. Commission of the European Communities; (iii) Comisión de las Comunidades Europeas. Decisión de 20 de junio de 2001. COMp/E-2/36.041/PO - MICHelin; (iv) Tribunal de Justicia (Sala Quinta). Sentencia de 15 de diciembre de 1994. Asunto C-250/92, Gottrup Klim (Rec. 1994, p. I-5641); (v) Comisión de las Comunidades Europeas. Decisión de 3 de julio de 2001. сомp/M.2220 - General Electric/Honeywell. 
Ahora bien, en relación con la extensión de esa independencia la misma fuente ha aclarado que la posición de dominio no se desvirtúa por la existencia de algún nivel de competencia. Por lo tanto, aunque el agente en cuestión no pueda determinar absolutamente las condiciones del mercado, se considera que tiene dominancia si puede generar una considerable influencia en tales condiciones y actuar sin estar sometido a ellas ${ }^{15}$.

Con fundamento en las fuentes citadas es posible establecer algunos criterios que, teniendo en cuenta la dinámica y las eficiencias que justifican la gestión colectiva, deben ser analizados con el fin de determinar si esa actividad económica puede ser considerada como indispensable para materializar los derechos de autor de los titulares. Los criterios son los siguientes:

- El número de usuarios interesados en utilizar las obras protegidas por el derecho de autor.

- El número de titulares de derechos de autor.

- Factibilidad de localizar a ambas clases de agentes.

- Certidumbre sobre la utilización del derecho. (Superintendencia de Industria y Comercio, 2016).

Ahora, la Superintendencia de Industria y Comercio (sIC) ${ }^{16}$ ha establecido que un agente económico tiene posición dominante cuando se encuentre en capacidad de "modificar significativamente y unilateralmente el precio, las cantidades ofrecidas o cualquier otra variable significativa para el mercado relevante, siempre que pueda mantener la modificación perdurablemente, porque la reacción de sus competidores, efectivos y/o potenciales, o de los consumidores, no sería suficiente para disuadirlo de realizar su conducta" (Superintendencia de Industria y Comercio, 2002).

En ese sentido, las decisiones de la Superintendencia de Industria y Comercio, así como la literatura especializada (Whish, y otros, 2011) también en (EC Competition Policy and IPR's, 2007), permiten concluir que la determinación de la existencia de las condiciones que determinan que un agente tenga dominancia exige analizar, entre otros, los siguientes aspectos: (i) las presiones competitivas ejercidas por los competidores existentes (las cuotas de mercado y niveles de concentración), (ii) las presiones competitivas ejercidas por la amenaza creíble de futuras expansiones de los competidores existentes o la entrada de competidores potenciales (expansión y barreras de entrada), (iii) las presiones competitivas

15 La concepción descrita es la que se ha seguido en el régimen de protección de la libre competencia económica de Colombia. En efecto, el artículo 45 del Decreto 2153 de 1992 define la posición de dominio como "[1]a posibilidad de determinar, directa o indirectamente, las condiciones de un mercado".

16 La sic ha desarrollado el concepto de posición de dominio en las siguientes resoluciones: 15653 de 10 de mayo de 2001 (caso Induga), 4285 de 11 de febrero de 2002 (caso Satena), 30835 de 14 de diciembre de 2004 (caso Setas), 33361 de 2011 (caso Bavaria), 53992 de 14 de septiembre de 2012 (caso Agua en Bloque), 4907 de 2013 (caso Gases de Occidente S.A. E.S.P.) y 53403 de 3 de septiembre de 2013 (caso Portabilidad). 
ejercidas por la fuerza o poder de negociación de los demandantes y, en fin, (iv) "otros factores que le permitan a la empresa actuar de manera independiente en el mercado" (Superintendencia de Industria y Comercio, 2013), entre los que se puede contar el que la empresa en cuestión, debido a las condiciones de contexto, se convierta en un agente indispensable para el desarrollo de actividades económicas por parte de otros participantes en el mercado (Tribunal de Justicia (sala tercera)) y (Monti, y otros).

En relación con los factores que determinan la configuración de la dominancia es importante resaltar, con fundamento en los pronunciamientos internacionales que han inspirado el desarrollo normativo del régimen colombiano, que "[l]a existencia de una posición dominante es, en general, el resultado de una combinación de diversos factores que, considerados aisladamente, no serían necesariamente decisivos" (Superintendencia de Industria y Comercio, 2013). En consecuencia, tales factores deben ser considerados conjuntamente (Kretschmer, 2002).

Evidenciados los factores que configuran la posición de dominio de un agente en un mercado determinado es pertinente hacer referencia a la caracterización que se debe hacer por parte de la autoridad de competencia del mercado en el que se puede llegar a presentar la dominancia.

\section{I. EL CONCEPTO DE MERCADO RELEVANTE PARA DETERMiNAR LA POSICIÓN DE DOMINIO}

La sIC debe realizar un estudio sobre el mercado relevante donde se puede presentar la posición de dominio. Dicha entidad ha señalado que para la definición del mercado relevante basta con la identificación de los bienes y servicios sobre los cuales recae la concentración y el ámbito territorial dentro del cual ejerce su actividad económica.

En efecto, sobre este particular la Superintendencia ha tenido oportunidad de precisar que del estudio económico que se realice debe establecerse el mercado producto y el mercado geográfico. Para el primero se debe establecer el bien o servicio que presta el agente económico dentro del mercado, para el segundo se debe establecer el ámbito geográfico en el que el agente desarrolla su actividad económica. Lo anterior significa que el mercado geográfico de las SGC será el territorio colombiano, pues si se presenta la explotación de una obra de la que sean administradoras en otro país, a través de los contratos de reciprocidad referidos en líneas anteriores, ejercerán el cobro de esa utilización.

En lo que se refiere al mercado producto, se necesitará determinar sobre qué derecho patrimonial y cuál modalidad de uso del mismo la SGC desarrolla su actividad, toda vez que dependerá de cada forma de utilización si la SGC ostenta o no posición de dominio.

Identificada la importancia de la determinación del mercado relevante para que la sIc pueda establecer la dominancia de una SGC o no, a continuación se describen 
casos nacionales e internacionales en los que se ha determinado por parte de la autoridad de competencia posición de dominio de las SGC.

\subsection{En Colombia la Superintendencia de Industria y Comercio HA DECRETADO LA POSICIÓN DE DOMINIO DE UNA SOCIEDAD DE GESTIÓN COLECTIVA}

La primera decisión de la sic que le atribuyó a una SGC posición de dominio fue en el caso Sayco, mediante la resolución de sanción n. ${ }^{\circ} 10150$ del 7 de marzo de 2017. En este caso el mercado definido por la autoridad fue, desde el punto de vista del mercado geográfico, Colombia, y desde el mercado producto: (i) la gestión colectiva del derecho de comunicación pública en la modalidad de establecimientos abiertos al público y (ii) la gestión colectiva del derecho de comunicación pública en la modalidad de conciertos.

Lo anterior obedece a un estudio realizado por la sic en el que determinó que la gestión de las mencionadas modalidades del derecho patrimonial de comunicación pública, por sus características propias, resultaba indispensable realizarla a través de la modalidad de gestión colectiva.

En este caso, la Superintendencia sancionó a Sayco por la infracción de los numerales 3 y 6 del artículo 50 del Decreto 2153 de 1992.

\subsection{Algunas decisiones de tribunales de competencia sobre la posición DE DOMINIO DE LAS SOCIEDADES DE GESTIÓN COLECTIVA}

Como se aprecia en decisiones de autoridades de competencia de varios países, la dominancia de una SGC está claramente establecida.

Ciertamente, coinciden en concluir que esas instituciones tienen posición de dominio, en el mercado del derecho patrimonial que corresponda, las siguientes decisiones relacionadas con una SGC:

a. España (sgAe, entre otras), Tribunal de Defensa de la Competencia. Resolución de 25 de enero de 2002, exp. 511/01 (Vale Music/sGaE) y Resolución de 27 de julio de 2000, exp. 465/99 (EGEDA). Comisión Nacional de la Competencia. Resolución de 9 de diciembre de 2008, exp. 636/07 (AGEDI), Resolución de 27 de julio de 2000, exp. 465/99 (AISGE).

b. Bélgica (SABAM), Tribunal de Justicia de las Comunidades Europeas. Decisión de 21 de marzo de 1974. Asunto 127/73. BRT II ECR-SABAM. ECR 51.

c. Alemania (Gema), Comisión de las Comunidades Europeas. Decisión de 2 de junio de 1971. IV/26760 - GEMA. 71(224/CEE) y Decisión de 4 de diciembre de 1981. IV/29.971 - GEMA Statutes. 82/204/EEC.

d. Francia (sacem), Comisión Europea. Decisión de 12 de agosto de 2002. Banghalter \& Homen Christo v. SACEM (Daft Punk) comp/C2/37.219. 
Si bien existe discusión y distintas posiciones sobre ciertos aspectos en los que se enfrentan los intereses de las SGC y los intereses de los titulares y usuarios, no hay discusión respecto de que, de acuerdo con decisiones internacionales en las que se sancionó por abuso de posición dominante, como:

a. Sabam, Tribunal de Justicia de las Comunidades Europeas. Decisión de 21 de marzo de 1974. Asunto 127/73. BRT II ECR-SABAM. ECR 51,

b. GEMA, Comisión de las Comunidades Europeas. Decisión de 2 de junio de 1971. IV/26760 - GEMA. 71(224/CEE) y Comisión de las Comunidades Europeas. Decisión de 6 de julio de 1972. IV/26.760 - GEMA. 72(268/CEE) y

c. SACEM, Comisión Europea. Decisión de 12 de agosto de 2002. Banghalter $\&$ Homen Christo v. SACEM (Daft Punk) COMP/C2/37.219,

de presentarse cualquiera de los siguientes supuestos se hace evidente el abuso de posición de dominio de una SGC:

- Que la sociedad de gestión colectiva obligue a los titulares a ceder todos sus derechos, sin posibilidad de elegir cuáles de esos derechos se ceden o no, so pena de no poder acceder a los servicios prestados por la sociedad de gestión colectiva.

- Que la SGC imponga cargas injustificadas a los titulares que previamente han cedido o licenciado derechos a la SGC puedan, si así lo desean, gestionar los derechos que no hayan cedido o licenciado a la SGC de forma individual.

En consecuencia, establecido que una SGC tiene posición de dominio, resulta necesario que esta actúen con mayor diligencia frente al régimen de libre competencia económica, toda vez que lo reprochable por la legislación no es que una SGC tenga posición de dominio sino que abuse de ella, contraviniendo así la prohibición de toda práctica restrictiva de la competencia, esto es, configurando el abuso de posición de dominio establecido en el artículo 50 del Decreto 2153 de 1992.

\section{Conclusión}

Para cumplir con el propósito de este escrito y presentar unos lineamientos sobre la gestión desarrollada por las SGC para que no incurran en la conducta reprochada por el artículo 50 del Decreto 2153 de 1992 -sobre abuso de posición de dominio-, a continuación se presentan las siguientes recomendaciones a tener en cuenta.

Sea lo primero anotar que, como se describe a lo largo del texto, la posición de dominio se enmarca en un mercado determinado, por esto, para identificar si una SGC ostenta dominancia o no es necesario establecer el mercado relevante. Lo anterior implica conocer el ámbito geográfico de acción de la sGC, que es nacional, 
en este caso referido a Colombia, y cuáles derechos patrimoniales y modalidades de ellos administra la SGC para conocer el mercado producto.

Lo anterior porque la posición de dominio de una SGC no se predica de todas las modalidades de utilización de un derecho patrimonial, sino de aquellas formas de explotación de la obra que resulta indispensables realizar a través de una SGC por sus eficiencias y su carácter de indispensabilidad.

Una vez identificado el mercado relevante y establecida la dominancia para ese mercado en específico, lo conveniente es prevenir cualquier abuso de esa posición.

En ese sentido, constituiría un abuso de posición de dominio por parte de las SGC incurrir en cualquiera de los seis supuestos contenidos en el artículo 50 del Decreto 2153 de 1992, a saber:

1. La disminución de precios por debajo de los costos cuando tengan por objeto eliminar uno varios competidores o prevenir la entrada o expansión de estos.

2. La aplicación de condiciones discriminatorias para operaciones equivalentes, que coloquen a un consumidor o proveedor en situación desventajosa frente a otro consumidor o proveedor de condiciones análogas.

3. Los que tengan por objeto o tengan como efecto subordinar el suministro de un producto a la aceptación de obligaciones adicionales, que por su naturaleza no constituían el objeto del negocio, sin perjuicio de lo establecido por otras disposiciones.

Si la SGC obliga a los titulares a ceder o licenciar todos sus derechos, sin posibilidad de elegir cuáles de esos se ceden/licencian o no, so pena de no poder acceder a los servicios prestados por la sociedad de gestión colectiva.

4. La venta a un comprador en condiciones diferentes de las que se ofrecen a otro comprador cuando sea con la intención de disminuir o eliminar la competencia en el mercado.

5. Vender o prestar servicios en alguna parte del territorio colombiano a un precio diferente de aquel al que se ofrece en otra parte de dicho territorio, cuando la intención o el efecto de la práctica sea disminuir o eliminar la competencia en esa parte del país y el precio no corresponda a la estructura de costos de la transacción.

6. Obstruir o impedir a terceros el acceso a los mercados o a los canales de comercialización.

Si la SGC impone cargas injustificadas a los titulares que previamente han cedido o licenciado derechos a la sociedad de gestión colectiva.

Descritas las prohibiciones establecidas en el régimen de protección de la libre competencia para el abuso de posición de dominio, la recomendación final es 
que las SGC sean conscientes de que tienen una posición de dominio y que, por lo tanto, deben tomar las medidas necesarias para no actuar en abuso de la misma.

\section{REFERENCIAS}

Anderman, Steven D. y Schmidt, Hedving, EC Competition Policy and IPR's, Cambridge University Press, 2007.

Azzopardi, Annalies, “Dominant Position': A Term in Search of Meaning”, en Christine A. Varney (ed.), The Cartels and Leniency Review, 2. ${ }^{\mathrm{a}}$ ed., Law Business, 2014.

Colombia. Corte Constitucional. Sentencia C-124 de 2013.

Colombia. Corte Constitucional. Sentencia C-509 de 2004.

Colombia. Corte Constitucional. Sentencia C-833 de 2007.

Colombia. Dirección Nacional de Derecho de Autor. Concepto 2-2007-2598 de $1 .^{\circ}$ de marzo de 2007.

Colombia. Superintendencia de Industria y Comercio. Caso Portabilidad. Resolución n. ${ }^{\circ} 535403$ de 3 de septiembre de 2013.

Colombia. Superintendencia de Industria y Comercio. Caso Satena. Resolución n. 4285 de 11 de febrero de 2002.

Colombia. Superintendencia de Industria y Comercio. Rad. 11-150526 (Sayco), Informe motivado, 2016.

España. Comisión Nacional de la Compentencia. "Informe sobre la gestión colectiva de derechos de propiedad intelectual", 2009.

Estados Unidos. Supreme Court. 1979. Broadcast Music, Inc. et al. v. Columbia Broadcasting System, Inc. et al. 441 U.S. 1, 1979.

Estados Unidos. United States District Court. 2014. Pandora Media, Inc. v. American Society of Composers, Authors and Publishers, Southern District of New York, 2014.

Ficsor, Minály, La gestión colectiva del derecho de autor y los derechos conexos, Ginebra, OMPI, 2002.

Kretschmer, M., "The failure of property rules in collective administration: Rethinking copyright societies as regulatory instruments”, EIPR, 24/3, 2002.

Lipszyc, Delia, Derecho de autor y derechos conexos, Buenos Aires, Unesco, 2006.

Merges, Robert P., "Contracting into Liability Rules: Intellectual Property Rigths and collective rights organizations”, California Law Review, vol. 84, 1996.

Merges, Robert P., "Contracting into Liability Rules: Intellectual Property Rights and Collective Rights Organizations", California Law Review, vol. 84, 1996, p. 1328.

Monti, Giorgio y Damian, Chalmers, European Union Law. Text and Materials, Updating Supplement, Cambridge University Press.

Monti, Giorgio, “The Concept of Dominance in Article 82”, European Competition Journal, vol. 31, 2006. 
Pérez Gómez Tétrel, Ana María, "Análisis económico de la gestión del derecho de autor", Revista La Propiedad Inmaterial, n. ${ }^{\circ}$ 15, Universidad Externado de Colombia, 2011.

Pérez Gómez Tétrel, Ana María, "Considering the Risk Dimension in the Administration of Copyright", Review of Economic Research on Copyright Issues, vol. 5, n. ${ }^{\circ} 1,2008$.

Pérez Gómez Tétrel, Ana María, El análisis económico del derecho de autor y del copyright, un estudio del equilibrio entre los intereses público y privado, Bogotá, Universidad Externado de Colombia, 2006.

Peritz, Rudolph, Competition policy and its implications for intellectual property rigths in the United States, Cambridge University Press, 2007.

PO-MICHELIN. COMPE-2/36.041/, s.l. : Comisión de las Comunidades Europeas, 20 de junio de 2001.

Porras, Antonio (ed.), "De las entidades de gestión de los derechos reconocidos en la ley", Endersa, Derecho Privado, vol. V.4B, 1995.

Tribunal de Justicia de las Comunidades Europeas, Hoffmann-La Roche \& Co. AGv. Commision of rhe European Communities. Asunto 85/76, 13 de febrero de 1979.

Tribunal de Justicia de las Comunidades Europeas. United Brands Company and United Brands Continental BV v. Commission of European Communities. Asunto 27/26, 14 de febrero de 1978.

Tribunal de Justicia, Sala Tercera. British Airways PLC v. Commission of ehe European Communities. Decisión de 15 de marzo de 2007.

Whish, Richard y Bailey, David, Competition Law, Oxford University Press, 2011. 